\title{
Protein microarray technology: Assisting personalized medicine in oncology (Review)
}

\author{
MONICA NEAGU ${ }^{1-3}$, MARINELA BOSTAN $^{1,4}$ and CAROLINA CONSTANTIN ${ }^{1,2}$ \\ ${ }^{1}$ Department of Immunology, 'Victor Babes' National Institute of Pathology, 050096 Bucharest; \\ ${ }^{2}$ Research Core of The Pathology Department, Colentina University Hospital, Bucharest 020125; \\ ${ }^{3}$ Faculty of Biology, University of Bucharest, 050095 Bucharest; ${ }^{4}$ Immunology Center, \\ 'Stefan Nicolau' Institute of Virology, 030304 Bucharest, Romania
}

Received April 23, 2019; Accepted June 11, 2019

DOI: $10.3892 /$ wasj.2019.15

\begin{abstract}
Among proteomics technologies, protein microarray, over the past last years, has gained an increased momentum in the biomarkers discovery domain. The characteristics of protein microarray, namely that it is a high-throughput tool, it provides a high specificity and only requires a minute amount of biological samples, render it a suitable tool for searching, quantifying and validating biomarkers in various pathologies. Protein microarray is based on the specific antigen-antibody reaction, such as any enzyme-linked immunosorbent assay, the specific reaction occurring on a miniaturized support (chip or slide), thus having the advantage of simultaneous evaluation of tens to thousands of molecules in small samples with a highly specific recognition for the detection system. In this review, we highlight the history of protein microarray technologies development and discuss this technology is stepping
\end{abstract}

Correspondence to: DrMonica Neagu, Department of Immunology, 'Victor Babes' National Institute of Pathology, 99-101 Splaiul Independentei, 050096 Bucharest, Romania

E-mail: neagu.monica@gmail.com

Abbreviations: ELISA, enzyme-linked immunosorbent assay; NAPPA, nucleic acids programmable protein array; SERPA, serologic proteome analysis; SEREX, serological analysis of recombinant tumor cDNA expression libraries; CTLA-4, cytotoxic T lymphocyte antigen 4; RPPA/RPPM, reverse-phase protein array/microarray; IPA, Ingenuity Pathway Analysis; TAA, tumor-associated antigen; NSCLC, non-small cell lung cancer; PD-L1, programmed cell death protein 1 ligand; MEK, mitogen-activated protein kinase kinase; COPD, chronic obstructive pulmonary disease; ARDS, acute respiratory distress; MMP, matrix metalloproteinase; HER2, human epidermal growth factor receptor 2; TMT LC-MS/MS, tandem mass tag liquid chromatography/tandem mass spectrometry; CCC, clear cell carcinoma; HGSC, high-grade serous carcinoma; IL, interleukin; ccRCC, clear cell renal carcinoma; AML, acute myeloid leukemia

Key words: protein microarray, proteomic, multiplexing, personalized medicine, biomarkers, diagnosis, oncology into the future. We present personalized medicine goals and discuss how protein microarray can aid in these goals, with an emphasis on several oncological diseases. We also discuss how protein technology has been used in diseases, such as lung, breast cancers, as well as in other diseases that, over the past last years, have taken advantage of this proteomic endeavor.

\section{Contents}

1. Introduction

2. Personalized medicine: A step forward in improving disease management

3. Protein microarray in oncology

4. Conclusions and future perspectives

\section{Introduction}

History of array technologies. Array tools were developed more than 25 years ago with the implementation of DNA microarray technology as a very accurate platform intended to determine mRNA expression for the concomitant determination of thousands of genes. One of the first studies, if not actually the first one on this subject, was published in 1995 when describing microarrays automatically printed with complementary DNAs on glass so that corresponding genes could be identified from samples. The use of low quantities of input sample due to the high density of the array, the detection of rare transcripts from only $2 \mu \mathrm{g}$ total mRNA was reported. In that seminal study, the expression levels of 45 Arabidopsis genes were measured using two-color fluorescence hybridization (1). Relatively soon, the necessity of DNA microarrays to evolve towards protein microarrays became obvious, as mRNA profiles were not perfectly matching protein expression (2).

Thus, although the number of human genes is in the order of tens of thousands, the protein synthesis system can express millions of protein types, these proteins being structurally and functionally inter-connected for maintaining tissues and cells homeostasis. With this finding, the scientific stage was open to the development of protein microarrays. Thus, protein microarrays were designed to cover the complex proteome machine 
and to pursue in the identification of protein functionality and interconnection. There are several types of protein microarrays, although the first type that was developed was based on specific antibody immobilization (3). Basically, it reproduced an enzyme-linked immunosorbent assay (ELISA) setup, having the advantage of highly specific recognition for the detection system. When technology could spot, in small quantities, specific antibodies without hindering their specificity and selectivity, conventional immunoassays turned to spotted arrays that allowed for multiple, specific and parallel detection of biomolecules from small amount of biological samples (3). The overall scheme of a protein microarray is presented in Fig. 1.

During its development and implementation in clinical units, this technology gained sensitivity and overall performance that led to its recognized validity. Further keeping the similarity to ELISA types, another design of protein microarray was developed. In this new version of protein microarray, immobilized purified proteins and not antibodies were deposited on the glass slides, and this new type was denominated as a functional array $(4,5)$. In this variant, various proteins can be spotted from a certain cell type, a group of cells or a tissue sample, or even an entire micro-organism can be placed on the slide (4-6). Functional microarrays can evaluate some key aspects in proteomics, protein functions, interconnection in protein-protein binding, metabolic/biochemical action, the association between a specific ligand and its receptor, the interrelation between an enzyme and its substrate, and can visualize immune-related protein(s) triggered by an active response (7).

Another design of protein microarrays is the reverse-phase type where total proteins from tissue/cell lysates or specifically fractionated tissue/cell lysates are spotted on the glass slides and their expression is hence quantified (8). Thus, in 2007, Speer et al published for the first time, this new protein microarray type. Their study demonstrated that in the need to depict functional alterations within the proteome, reverse-phase protein microarray can detect altered cellular protein molecular networks and cell signaling pathways associated to human diseases, particularly cancer. Tumorigenesis is based on protein molecular networks alteration leading to disrupted cell signaling pathways, uncontrolled proliferation, drug resistance, enhanced mobility and the adaptation to a new microenvironment in the metastatic processes (8).

From the very beginning, this new type of protein microarray was intended to depict the pathology molecular profile, as it can provide 'functional read-out of cell signaling networks or pathways for an individual patient'. The assertion of these authors is of outmost importance in the clinical context of personalized medicine where patient stratification for the most efficient therapy is the ultimate goal (8).

In the biomedical research field, protein microarray is increasing its utility in assisting treatment efficacy by evaluating for example certain apoptotic markers (e.g., BCL-2, BCL-X and BAD) upon the application of various therapies (9), or assessing transcriptional activity in cells that were subjected to therapy (10). Thus, protein microarray has developed into a proteomic tool that can deliver high-throughput data for revealing novel therapeutic targets. There are currently three main types of protein microarrays based on their reaction principle and application: Analytical or antibody, reverse-phase and functional protein microarrays (11).

Among all the developed variants, in oncology, the antibody array type is the preferred one, where it applies discovery and quantification to biomarkers. The antibody array layout has high versatility and reproducibility (12). By contrast, the reverse-phase array format has a lower standardization potential and multiplexing possibilities and it is prone to cross-reactivity, as all the proteins harbored by complex samples are investigated at once (13).

These different protein microarray formats can accommodate a variable number of spots, and can be used to successfully aid personalized medicine. Protein arrays can encompass $>1,000$ elements per array and these ones are termed 'high-density arrays' (14). Such arrays are used for the identification of new proteins or novel protein/protein interactions. Protein libraries or even unknown elements can be spotted on the array and various biological samples can be used. The detection is insured by antibodies that are directly labeled with a fluorophore. Protein-protein binding events can be also detected in these formats using specific antibodies $(15,16)$. Reverse-phase protein arrays use a lower range of samples, up to hundreds, to identify a small number of antigens. This format can use cell lysates, micro-dissected tissues/cells, and biological fluids, such as plasma and serum. The detection antibody labeled with a fluorophore would visualize the reaction between capture antibody and analyte of interest from a biological sample (17).

'Low-density arrays' with 9-100 elements per array are antibody arrays that are used for the quantitative detection of proteins in cells, tissues and biological fluids samples. The density of the antibody array is constantly expanding due to the generation of high-affinity antibodies (18). Thus, specific antibodies are arrayed and they will capture antigens from samples. The identified antigen will be directly labeled with a fluorophore or a secondary antibody will be used for detection. This process is known from the sandwich ELISA assay (19). Table I presents an overall view of the classification of major protein microarray formats.

Protein microarrays: 'Ongoing future'. Following an investigation of the PubMed database using key words, such as 'Protein microarray' AND 'Personalized Medicine' and 'Protein microarray' AND 'Personalized Medicine' AND 'Cancer', we obtained some very interesting facts on this issue (Fig. 2). First of all, an increase in the number of publications can be observed, beginning from the year 2012, and subsequently maintaining an accelerated trend up to 2017, when the number of publications decreased. Secondly, the publications for protein microarrays in personalized medicine has an identical trend for its use in cancer, this trend showing that the majority of publications in personalized medicine are based on this type of human pathology.

Tumorigenesis is a multi-proteomic complex process in which the transformed cells will crosstalk with immune and stromal cells, favoring tumor development, dissemination, neo-angiogenesis, enhance immune-escaping processes, epithelial-to-mesenchymal transition, invasion and multi-drug 


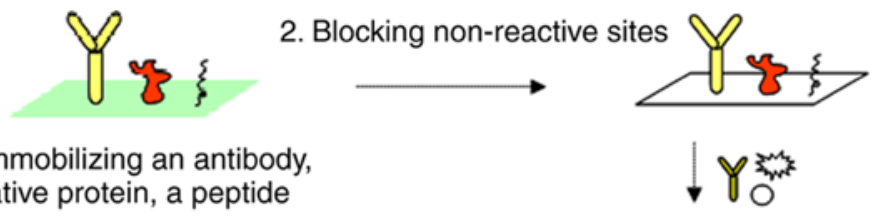

1. Immobilizing an antibod
a native protein, a peptide

3. Sample incubation

a. No labeling (MS, SPR, AFM)

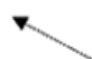

\section{Sample incubation}

5. Detection of binding

b. Specific labeling

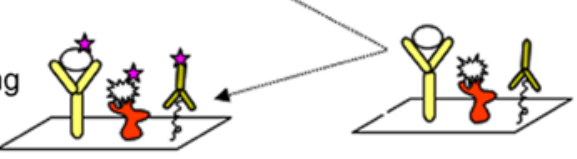

4. Specific binding

Figure 1. The general outline of any protein microarray workflow. 1) Immobilizing an agent (only for customized protein microarray slides); 2) Blocking the non-reactive sites; 3) Sample incubation on specific slide; 4) The specific binding between an analyte from the sample and its corresponding antibody attached on the slide; 5) Detection step that can be (a) without any labeling through e.g., mass spectrometry (MS), surface plasmon resonance (SPR), atomic force microscopy (AFM) or (b) with a fluorescent dye (6).

resistance (29). All these processes rely on direct contact through protein-based receptors and ligands or soluble protein molecules (e.g., growth factors, cytokines and chemokines). Recently nucleic acids structures, such as microRNAs encompassed in extracellular vesicles are also reported as communication avenues (30).

Proteomics represents the large-scale study of proteins, depicting structure, interactions and functions. Amidst proteomics domain protein microarray stands in these high-throughput approaches (31).

Protein microarray is involved in deciphering cellular differentiation, transformation, angiogenesis, tumorigenesis and metastasis. Due to its development, it can detect alterations in protein expression levels, identify post-translational modification, mRNA events, and identify molecular networks triggered by therapeutic approaches, but also by environmental factors. The future of protein microarray is continuously expanding, so that profiling the cancer signaling network, personalizing therapy and improving diagnosis and prognosis would take a step forward (32).

The accelerated future of protein microarray relies on several domains: Improving its technicalities approaches with improved sensibility and specificity, expanding on areas, such as cell-free microarrays and immunoproteomics and last, but not least, develop the bioinformatics technologies that actively sustains this type of technology (32). Proteomics techniques would provide information on the proteins and peptides, and the dynamics of their interconnections. Hence, a number of methodological developments and innovations have been reported over the past decade. Protein networks are best studied using nucleic acid programmable protein array (NAPPA). Following its design a decade ago (33), it is evolving with high accuracy, increased reproducibility, throughput and flexibility in diagnostic and therapeutic applications. NAPPA is an essential tool that deciphers functional proteomics along with protein-protein interaction (34). In NAPPA microarrays, the extra-well fluorescence automated acquisition was reported. Different approaches have been used to identify spots with extra-well fluorescence (rings) in the microarray images and the reported system, would identify in a significantly more rapid manner, than any human would, this extra-fluorescence, while maintaining high performance for microarray image analysis (35).

Cell-free protein microarrays are an array variant which display fresh proteins, avoiding storage and denaturation. In basic and translational research, this is a format that is steadily increasing in order to identify protein-protein interactions, pathogen-host associations, post-translational modifications, and antibody-type biomarkers (36). Displaying actually naturally-folded proteins has increased the risk of spot-to-spot crosstalk due to protein diffusion during expression. Thus, recently, the multiplexed nucleic acid programmable protein array (M-NAPPA) was reported. This improved technology increases the number of displayed proteins through five different gene plasmids in a single printed spot. Due to this improved technology, M-NAPPA, an ultra-high density proteome microarray could be done having $>16,000$ proteins per slide. This multiplexing has improved features, and is a new protein microarray for high-throughput translational research (37).

Immunoproteomics is actually a recent extension of the proteomics domain study of immune-related proteins and peptides (38) and encompasses the future of protein microarrays. Immunoproteomics began over 30 years ago for the identification of tumor antigens, and is one of the main goals in oncology (39). Proteins released by the tumor trigger an immune response activating antigen-specific T and B cells. An efficient immunotherapy destroys tumor cells, cellular proteins are released and $\mathrm{T}$ and $\mathrm{B}$ lymphocytes are activated. As new antigens are spreading, auto-antibodies are generated, and thus auto-antibodies against tumor antigens can be a measure of efficient immunotherapy (40).

From the early 1980 s, several immunoproteomics methodologies were approached. Thus, serologic proteome analysis (SERPA), accompanied by serological analysis of recombinant tumor cDNA expression libraries (SEREX) aided by protein microarrays were lately exploring tumour antigens using specific antibodies (41). SERPA can screen antibody profiles in patient serum using proteins appended to a membrane and is a highly used immunoproteomics workflow. SEREX actually discovered the first cancer testis antigen, NY-ESO-1 (42). SERPA and SEREX have their limitations, mainly due to the 
Table I. Classification of protein microarray types (6).

\begin{tabular}{|c|c|c|c|}
\hline Classification criteria & & Type & (Refs.) \\
\hline \multirow[t]{3}{*}{ Immobilized structure } & Direct & Standard: Recombinant purified proteins are immobilized & $(20,21)$ \\
\hline & & Analytical: Antibodies are immobilized & $(5)$ \\
\hline & Indirect & Reverse phase protein microarray (total or fractionated cellular lysates) & $(8)$ \\
\hline \multirow[t]{5}{*}{ Determined parameter } & Abundance & Capture & $(22)$ \\
\hline & & Indirect & $(23)$ \\
\hline & Functional & Protein in situ arrays & $(24,25)$ \\
\hline & & In situ puromycin arrays & (26) \\
\hline & & Nucleic acids programmable protein (NAPPA) array & $(27,28)$ \\
\hline
\end{tabular}

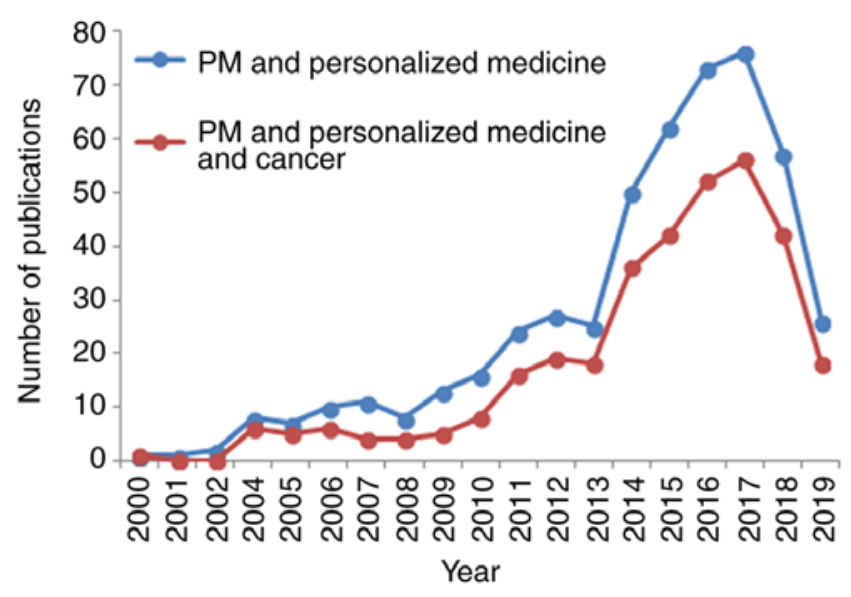

Figure 2. Number of publications on the PubMed database retrieved using key words, such as 'Protein microarray' (PM) AND 'Personalized Medicine' and 'Protein microarray' (PM) AND 'Personalized Medicine' AND 'Cancer'

proteins on the SEREX membrane expressed by the library expressed in bacteria, expression levels that cannot cover human post-translational protein modification. Furthermore, protein microarrays have additional characteristics, such as thousands of pure proteins that are immobilized on a glass surface. For example, ProtoArray ${ }^{\circledR}$ (functional array), can analyze concomitantly thousands of proteins from a serum sample. These types of protein microarray have developed a new domain known as seromics (43). Tumor-associated antigens and their generated antibodies are the major proteins that can be detected using proteomics (44). The use of SEREX tumor antigens that elicit a high IgG antibody titer in sera from patients diagnosed with different types of cancer has been established (45).

Cancer immunotherapies, entering clinical management, have taken advantage from the research stage of protein microarray technology. Auto-antibodies generated during therapy were correlated with tumor progression in patients diagnosed with prostate, lung, ovarian and breast cancer (46-48). Sipuleucel-T therapy, actually the first approved adoptive cell therapy, is based on an antigen spreading that leads to an immune response and an improved overall survival (49). In prostate cancer, cytotoxic T lymphocyte antigen 4 (CTLA-4) blockade generates a broad antibody response in therapy responsive patients (49) and moreover, this response is directed to proteins that were reported as mutated in prostate cancers (50).

The advantages brought by protein microarray technology include a reduction in biological sample volume, high sensitivity/specificity and large data generation. The sensitivity and specificity of a protein microarray with 329 proteins gives a $94 \%$ concordance with standard ELISAs (51). As in gene microarrays, there are several standard methodologies that should be followed in order to obtain accurate data, such as proper biological sample collection, correct storage and standard laboratory procedures that avoid inter- and intra-assay variation, thus reinforcing data reproducibility (32).

In addition, bioinformatics specific for protein microarray needs specific development in order to process large amounts of data arising. Analyzing protein microarray data implies the following steps: Data acquisition, data pre-processing, visualization, differential analysis, computational annotation and network analysis $(52,53)$.

The use of detection antibodies provides very good sensitivity and specificity for protein identification, but it has also some limitations. Antibodies are also proteins and thus, any disturbances that will alter their structure (e.g., $\mathrm{pH}$ and temperature) will affect the binding specificity. Antigen-antibody interaction has complex kinetics, and again, any conditions that would influence this interaction can hider both the specificity and affinity of the bonding (54). The selection of the 'perfect antibody' is another limitation of the technology, as it should possess strong affinities and specificity displayed for specific proteins, more so if intimate modifications of the proteins are to be identified, e.g., phosphorylation, glycosylation or proteolysis-related compounds. When the specific protein microarray has also a quantification need, hundreds of supposed antibody-antigen complexes are established having independent affinity parameters. Protein concentrations in the samples, whether biological fluids, cell/tissue lysates, can have hundreds of fold different scale. Therefore, detection needs to cover concentrations over many orders of magnitude $(53,54)$. An overview of major advantages and disadvantages for the most used protein microarrays variants $(55-57)$ is presented in Table II.

With the advent of the Human Genome Project depicting just $>20,000$ genes, it became evident that a new initiative should be covered at the proteome level. Hence, 2010 was the year for launching the Human Proteome Project. The 
technological strategy to cover this huge scientific endeavor implied 'three working pillars': Mass spectrometry, arrays and bioinformatics tools. If the first two pillars were based on the advantages that quantitative mass spectrometry and protein capture with antibodies brought a decade ago, the third one actually was based on the global exchange of databases and the availability of large primary data (58-59). This huge on-going project has driven, apart from the enormous scientific gain, a series of proteomics and bioinformatics methods. Hence, shotgun and selected reaction monitoring (SRM)/multiple reaction monitoring (MRM)-based proteomics were developed. The combination of different omics technologies has led to the development of intermingled domains, such as epitranscriptomics (60), proteogenomics, metabolomics and so on (61). Moreover, the Human Proteome Project has contributed to the clinical need for more targeted/individualized therapies, laying the foundation for the development of personalized medicine. Although fundamental research purposes prevail in the development of array platforms, there is a recent increasing trend in clinical research, diagnostics and even industry applications.

\section{Personalized medicine: A step forward in improving disease management}

Personalized medicine intends to unveil the molecular mechanisms of disease onset and to integrate it with individual genomics/proteomics/metabolomics profiles in order to define the most suitable drugs and provide a prognosis of the disease outcome (62). There are several fields that must be accomplished when dealing with the personalized medicine domain. First, a proper biomarker characterization for predicting the outcome and correct diagnostic markers must be identified, secondly it has to evaluate the perfectly matched therapy regimen and last, but not least, monitoring the disease and therapeutic efficacy come to complete a personalized approach. All these items can be evaluated by omics techniques (62).

'Diagnostic biomarkers' would be represented by protein expression from signaling pathways accompanied by key mutations. These markers will indicate the best drug(s) that will offer the optimal response in therapy-related decisions. 'Prognostic biomarkers' would encompass somatic germline mutations, epitranscriptomics modifications, miRNAs patterns and circulating tumor cells, and would thus determine disease outcome (63).

In personalized medicine, there is a clear necessity to identify the network comprising the genome-transcriptome-proteome patient profile. In oncology, it was estimated for 2018 , that $>1.5$ million new cases of cancer would be registered in the US, while there would be over half a million cancer-related deaths. According to the Annual Report, the most common types of cancer will be 'breast cancer, lung and bronchus cancer, prostate cancer, colon and rectum cancer, melanoma of the skin, bladder cancer, non-Hodgkin lymphoma, kidney and renal pelvis cancer, endometrial cancer, leukemia, pancreatic cancer, thyroid cancer and liver cancer'. Moreover, cancer incidence could achieve pandemic levels by the year 2025 (64).

Therefore, a complex approach in health needs high-throughput technologies that can sustain personalized medicine. Developing the two-tiered health system (65) to two-tiered personalized medicines is a demanding desiderate in oncology. The implementation of omics facilities in clinical practice is warranted in order to offer effective personalized medicine to the patient. However, in order to for this to be accomplished, there are several draw-backs that first need to be resolved, such as the high costs of implementation, differences between data generation and the capacity to analyze large amounts of data, the existence of multidisciplinary teams and global economic relevance (66).

Protein microarrays paving the way for personalized medicine. Personalized medicine intends to stratify patients as per disease subtype, risk factors, evaluated prognosis and/or treatment response. An interdisciplinary effort is needed between several specialties, physicians, data scientists and health insurance systems to provide un-biased advantage to clinical practice (67).

As stated in the 'Introduction', DNA arrays can analyze the whole transcriptome and can screen myriads of single-nucleotide polymorphisms, allowing further correlations of gene expression with disease progression. These analyses of disease-specific mutations can lead to setting specific therapies in accordance with their gene profiles. However, a more direct correlation with disease development is established by protein function, regulation and abundance. Driving the development of the disease protein concentrations within an organ, tissue, or cell can pinpoint an abnormality. Thus a patient's genotype can provide information regarding a particular stratification of the disease; however, the protein particularities, abundance, localization within the tumor cell/tissue and so on, would follow and describe the actual disease stage and would orient/personalize the therapy. Thus, personalized medicine would cover: 'Right patient/target, right diagnosis, right treatment, right drug/target, and right dose/time' (68). This goal can be achieved, however, only by combining genomic knowledge with traditional clinical approaches, the patient's personal medical and family history, and relevant clinical data, such as imaging and in vitro diagnostics results (69). Profiling using protein microarrays, can be efficiently applied in biomarker discovery, validation and diagnosis, and can aid personalized medicine (69). All the pathways that are deregulated in tumorigenesis and that are the result of genetic alterations accumulation can be, at least theoretically, therapeutic targets in oncology. The real-life efficacy of such molecular therapeutics is highly variable among individuals; thus, minute details of such differences can be identified. Reverse-phase protein array/microarray (RPPA/RPPM) can precisely map active molecules in each patient. This identification is essential for the optimization of therapy. RPPA is an antibody-based highly quantitative proteomic technology, used for profiling the expression and modification of signaling proteins, mainly in low-abundance analytes cases. Clinical trials are using RPPA technology molecular-targeted therapeutics (70); some of have not yet been completed (https://clinicaltrials. gov/ct2/show/NCT01042379), while others are already closed and waiting for the results of the evaluation (https://clinicaltrials.gov/ct2/show/NCT01023477, https:/clinicaltrials. gov/ct2/show/NCT01074814). For example, in breast cancer, which is a heterogeneous disease with various histological and 
Table II. Advantages and disadvantages in using the major protein microarray types in personalized medicine.

Main type of protein array method Analytical/antibody/indirect labeling (sandwich)

Reverse-phase/direct labeling

- Assess multiple analytes simultaneously

- Biomarker discovery

- Low sample consumption

Advantages

Disadvantages
- Could be arrayed as semi-quantitative or quantitative formats

- Improved specificity by using two specific antibodies (for capture and detection)

- Requires two specific antibodies for each target from sample of interest

- Still a challenging task to multiplex related to the feasibility of designing arrays with thousands of analytes
- Compares patterns of two analytes with different fluorophores

- Rapid screening of many different samples

- Specific antibodies could give cross reactions and hence could provide false results

- Rare analytes from biological samples may not be caught

- Relatively high production costs molecular variants, personalized medicine has become a major goal for patient management over the past years. In this type of cancer, molecular profiling and genomic analysis based on array technologies have led to the discovery of targeted drug therapies (71).

Using a designed RPPA, personalized therapy was intended to search for the most effective drug. Thus, drug sensitivity can be predicted in this system based on the quantitative profiles of protein expression (72,73). Signaling transduction pathways that trigger oncogenesis can also be depicted by proteomics profiling and these particularities can lead the option for personalized therapy $(74,75)$.

To increase the quantization sensitivity of RPPA $(76,77)$ labeling was reported to be far more accurate when using quantum dots (Qdots). Briefly, sample lysates are used for serial dilution and then immobilized on the array. Primary and secondary antibodies detect the immobilized proteins, and the reaction is further detected by Qdot assay. Qdot is actually a fluorophore with a nanometal structure that develops a clear linear signal, photo-bleaching from the organic fluorophores (78-80). In this form, RPPA would detect post-translational modifications, such as phosphorylation, modifications that are seminal for depicting intracellular events related to drug sensitivity (72).

Another recent version of protein arrays was published, bringing new information to personalized medicine. Thus, antibody co-localization microarray (ACM), avoids some draw-backs from classical protein microarrays (e.g., reagent-induced cross-reactivity), as detection antibodies are printed atop of their individual capture antibodies. Several parameters are improved, and therefore low volumes of sample and hence, low quantities of reagents are used. In this manner, up to 108 different protein-targets can be measured in hundreds of samples, displaying high specificity and sensitivity (81).

As protein microarrays are furnishing a plethora of records, several systems for data analysis have been reported. Ingenuity Pathway Analysis (IPA) is the most commonly used software for protein microarray data exploration (54). This software links to published database and finds function(s) and pathway(s) for microarray analysis. It can be used to integrate complex data from gene expression, microRNAs, single nucleotide polymorphisms (SNPs) and protein microarray (82). IPA ranks the genes and the encoded proteins as plausible candidate biomarker(s), identifies if a particular gene/protein can be detected in various tissues and/or other body fluids, it selects the relevant parameters for a specific biomarker discovery and shows possible links to a specific disease/biological process; moreover, it generates a list of unique markers to one treatment or disease, or reveals markers common for different diseases/therapies. Practice has shown that improvements in data processing systems are warranted; thus, recently reported Protein Microarray Analyzer software has several improved tools, as shown in Fig. 3.

To identify tumor-associated antigens (TAAs), antibody response and new antigen discovery other software were specifically developed for protein microarrays used in seromics, namely Prospector, LIMMA package, PAA package and Spotfire package $(49,50,53,83)$.

The newest intervention of protein microarray technology was reported for the revolutionary immunotherapies that were recently approved. New combinations of therapies are tested in pre-clinical settings. In mutant Kirsten rat sarcoma (KRAS) and tumor protein 53 (TP53) (KP) mouse models of non-small cell lung cancer (NSCLC), combinations of anti-programmed cell death protein 1 ligand (PD-L1), anti-CTLA-4 with mitogen-activated protein kinase kinase (MEK) inhibitors decreased tumor growth and metastasis. RPPA analysis and flow cytometric analysis of the tumors revealed an enhanced expression of Tregs and CTLA-4. Combining anti-CTLA-4 and anti PD-L1 with MEK inhibitor in a mouse model with good disease evolution has driven a phase I/II clinical protocol in humans that is now undergoing regulatory revision and enrollment began in 2019 (84).

\section{Protein microarray in oncology}

One of the main target domain of personalized medicine is the field of oncology, where the number of pre-clinical models and 


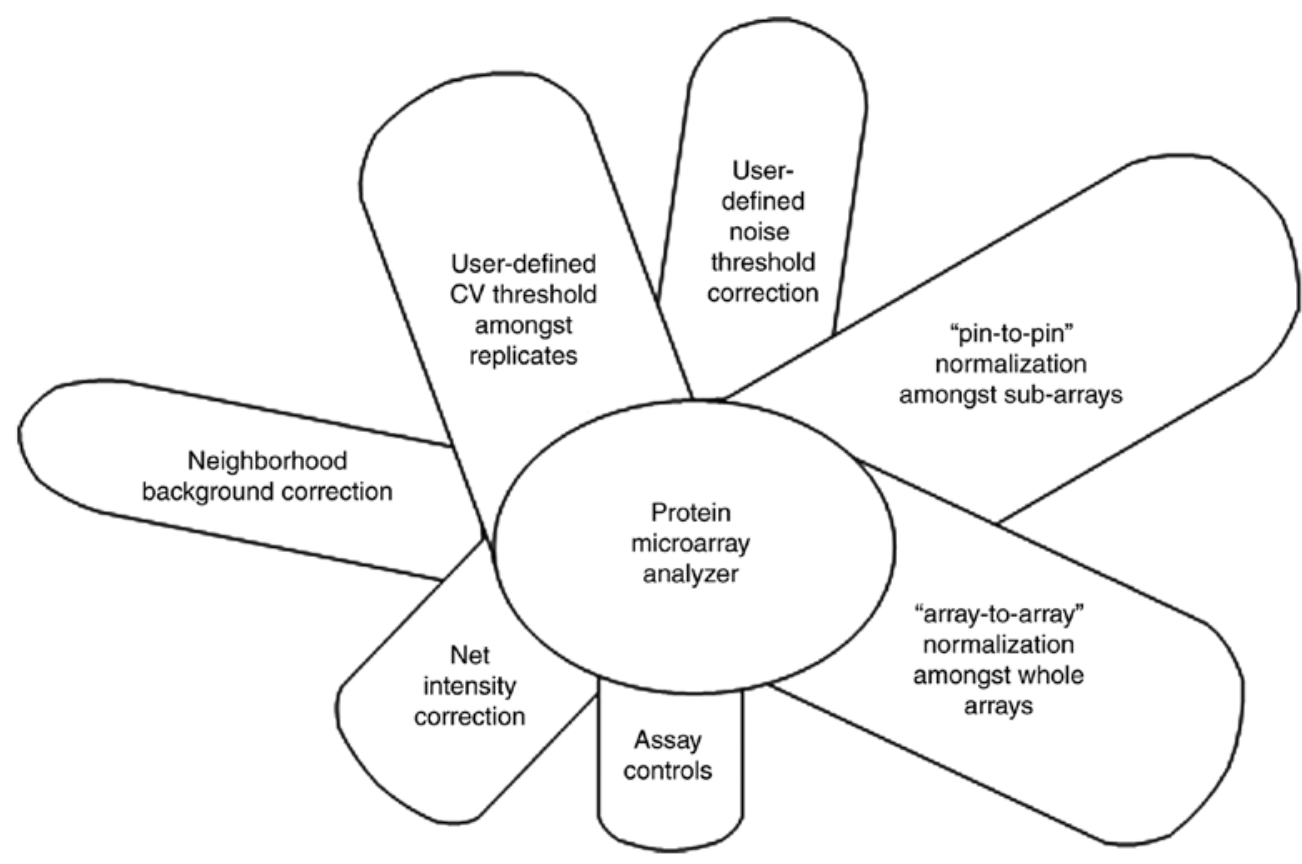

Figure 3. Protein Microarray Analyzer software characteristics.

clinical trials has increased over the past years (85); thus, new proteomics technologies have been put to use in acknowledging proteomic/genomic/transcriptomic/metabolic individualities (71). In this section, we will comment on the most frequent human pathologies that entail protein microarray technology in order to come one step closer to personalized therapy.

Personalized medicine through protein microarray in pulmonary disease. Pulmonary diseases infer a large range of biological samples, from sputum, to pulmonary epithelial lining fluid, bronchoalveolar and nasal lavage fluids, to exhaled breath condensate, and finally, blood plasma/serum (86). Due to this extended array of samples, the proteins that can be identified in these diseases comprise a huge span of molecules in terms of types and concentrations. Various proteomics technologies have been used; thus, PRoteomics IDEntifications (PRIDE) of the European Bioinformatics Institute has issued, over the past years, a database, containing $>70,000$ assays (87). In a previous study, the analysis of plasma from $>100$ children diagnosed with asthma evaluated for proteome patterns demonstrated that in comparison to the controls, there were 3 overexpressed proteins [chemokine (C-C motif) ligand 5 (CCL5), hematopoietic prostaglandin D2 synthase (HPGDS) and neuropeptide $\mathrm{S}$ receptor (member of the $G$ protein-coupled receptor 1 family) (NPSR)] with potential to be used as biomarkers (88).

Sputum proteomes from lung diseases [e.g., asthma and chronic obstructive pulmonary disease (COPD)] have been proven to exhibit enhanced concentrations of various other proteins, such as calgranulin A and B (89). Lung tissue from non-smokers compared to smokers and COPD, has been shown to have a different proteome, in which cathepsin D (CTSD), dihydropyrimidinase like 2 (DPYSL2), transglutaminase 2 (TGM2) and tripeptidyl peptidase 1 (TPP1) are differently expressed $(90,91)$.

In acute respiratory distress (ARDS), drug development and biomarkers to prognosticate the disease are crucial (92).
Deregulated cellular pathways leading to inflammation and epithelial injury have been revealed in ARDS. In lung tissue, as well as in biological fluids, several proteins have been found to be overexpressed, such as apolipoprotein A1, hemoglobin $\alpha$ and hemoglobin $\beta$ (93), osteopontin, matrix metalloproteinase (MMP)7, CXCL7, chemokine (C-X-C motif) ligand 7 (CXCL7), chemokine (C-C motif) ligand 18 (CCL18) and eosinophil- and neutrophil-derived proteins (94). However, there are still no validated markers available for subclassifying patients $(92,95,96)$. Therefore, subgroups of patients displaying particular molecular and clinical parameters could be identified using integrative omics data that will be required to accelerate personalized medicine upcoming in pulmonary diseases (97).

Lung cancer is the primary cause of cancer-related mortality in the United States, with a low 5-year survival rate of 18\%; therefore, this type of disease is an important subject in the light of personalized medicine. In a recent study, apart from the genomic profiling of lung cancer cell-surface markers, protein microarray was used to validate the high expression of the six selected markers that were identified in all the analyses [carbonic anhydrase 9 (CA9), carbonic anhydrase 12 (CA12), cancer/testis antigen 83 (CT83; also known as CXorf61), G protein-coupled receptor 87 (GPR87), LY6/PLAUR domain containing 3 (LYPD3) and solute carrier family 7 member 11 (SLC7A11)], these factors being associated with a worse survival. Hence, these markers could be further used for personalized care in patients with lung cancer (98). In lung cancer therapy, the identification of immune-checkpoints before therapy commences is a recent goal of personalized medicine. Tissue arrays and multiplex immunofluorescence have been used to evaluate 25 different types of immune-checkpoints and neoantigens. A recent study demonstrated that in lung therapy, protein-protein interaction and thorough intracellular signaling pathway mapping can reveal immune-checkpoint nodes that are associated with positive outcomes of the administered therapy (99). 
Personalized medicine in breast and ovarian cancers using protein microarray. As breast cancer is the second leading cause of cancer-related mortality among women worldwide, research has advanced at an accelerated pace. Over the past ten years, significant assessments, such as cytogenetics, SNPs and gene expression arrays, copy number variation and DNA methylation, have aimed to divide breast cancer types on a genetic basis. Apart from the genomic profiles, proteomics has begun to 'take the battlefield'. Thus, ten years ago, a comparison of proteomics profiling of invasive ductal carcinoma and normal tissues demonstrated that out of the 160 proteins/phospho-proteins tested, 56 were differentially expressed [e.g., Twist family BHLH transcription factor (Twist), Fas cell surface death receptor (Fas), proliferating cell nuclear antigen (PCNA), phosphatase and tensin homolog (PTEN) and cyclin B1]. These proteomics profiles distinguished tumor tissue from normal tissue with a $96 \%$ accuracy (54).

Recent proteomics studies have focused on drug-induced signaling events that would trigger a process that is of seminal importance to clinical application, namely acquired drug resistance. Following treatment with agents targeting human epidermal growth factor receptor 2 (HER2), phosphoproteome of the tumor cells was quantified using tandem mass tag liquid chromatography/tandem mass spectrometry (TMT LC-MS/MS). The activation of kinases families (e.g., serine/threonine and tyrosine kinases) following treatment was identified by peptide Chip array. Using these proteomics approaches, a common adaptive kinase response process was reported, involving the activation of the focal adhesion kinase 1 (FAK1), protein kinase $\mathrm{C}-\delta$ (PRKCD) and ephrin $(\mathrm{EPH})$ family receptors. These approaches bring into the light individual networks that can be activated when acquiring resistance to HER2-targeted therapies (100).

In the same area, an experimental model was recently reported, in which $\mathrm{HR}^{+} / \mathrm{HER} 2^{+}$patient-derived xenograft (PDX) were established in order to individualize HER $2^{+}$breast cancer therapies. Transcriptomic and proteomic profiling were used (RNA sequencing and RPPA) for establishing the molecular particularities of the PDX models. The reported results revealed that apart from standard trastuzumab therapies, the combination with the dual mammalian target of rapamycin (mTOR) complex inhibitor impeded tumor growth. Thus, this study opens the door for personalized medicine clinical trials (101).

From lysates obtained from samples of breast cancer tissues, a personalized medicine protocol was recently reported using RPPA. This protocol could be used for the pharmacodynamic effects of standard therapies in various molecular subtypes (102).

Drug resistance in breast cancer is a therapeutic domain where protein microarray can bring new information. Chemotherapy-resistant breast cancer stem cells (CSCs) were analyzed with protein arrays and the paclitaxel-resistant phenotype was associated with the overexpression of several proteins, such as growth factors, MMP proteins, Frizzled proteins and interleukin (IL)-23 (103).

Ovarian cancer also has a large array of subtypes, serous, clear cell, endometrioid and mucinous epithelial ovarian carcinoma, all these having various chemotherapeutic sensitivities. Clear cell carcinoma (CCC) has high rates of recurrence
Table III. Signaling pathways that are enhanced in ovarian clear cell carcinoma in comparison to other sub-types (108).

\begin{tabular}{ll}
\hline Pathway & \multicolumn{1}{c}{ Proteins } \\
\hline mTOR & PI3K/AKT \\
VEGF & HIF-1 $\alpha /$ VEGF \\
HNF-1 $\beta$ & HNF-1 $\beta$ \\
IL-6 & IL-6/STAT3 \\
MET & Ligand hepatocyte growth factor
\end{tabular}

mTOR, mammalian target of rapamycin; VEGF, vascular endothelial growth factor; HNF-1 $\beta$, hepatocyte nuclear factor $1 \beta$; IL-6, interleukin-6; MET, mesenchymal epithelial transition factor receptor; HIF- $1 \alpha$, hypoxia-inducible factor $1 \alpha$; STAT3, signal transducer and activator of transcription 3 .

associated with low chemosensitivity. Using RPPA, possible protein biomarkers have been investigated in patients with CCC and in high-grade serous carcinoma (HGSC). Thus, HER2 and PD-L1 expression levels were higher in patients with CCC, and aurora kinase A (AURKA) and PD-L1 were associated with CCC chemoresistance. The reported differences would lead to new candidate target drugs (104).

In CCC, various activating pathways have been reported, yet again, possible personalized drug targets (105). Proteins appending to various networks (Table III) $(106,107)$ were recently identified using RPPA. For example, 11 out of 117 proteins identified from CCC samples were appending to different signaling networks in comparisons to other ovarian cancer samples, giving ground to the further development of personalized therapy in this particular type of ovarian cancer which is difficult to treat (108).

Other types of diseases taking advantage of protein microarray technology in personalized medicine. In clear cell renal carcinoma (ccRCC), which is the most frequent renal cancer type, it is assumed that one third of patients would progress after surgery. Therefore, establishing molecular patterns that would stratify patients would significantly improve survival. In vitro cultures established from patient specimens have been used to develop orthotopic xenograft tumors in animal models. RPPA was used to evaluate the proteome in tumor cells and it was shown that tumor-propagating cells had clear altered kinase cascades, alterations that were associated with stage, the angiogenesis level and mTOR pathways. Testing in vitro and in vivo pharmacological action on ccRCC tumor cells can bring a personalized screening for therapies in patients, hence personalizing the therapy. Accordinly, only a high-throughput profiling, such as the one provided by RPPA could cover all the triggered pathways (109).

Custom RPPA was used to establish the protein profiling in pediatric acute myeloid leukemia (AML) bone marrow samples in comparison to normal samples. Protein functional groups and protein clusters identification has shown that there are 12 protein clusters that can stratify AML patients into 8 protein signatures. The identification of particular protein signatures creates the premises for specific combinations of 
therapies with increased therapeutic efficacy (110). Thus, in a phase II clinical trial, the efficacy and safety of a combination of the pan-AKT inhibitor (GSK2141795) and the MEK inhibitor, trametinib, in RAS-mutated AML were examined. Using RPPA the phospho-flow analysis assessment of the MEK and AKT pathways was performed in patients receiving this combination of drugs (111).

In colorectal cancer the immunoproteomics endeavor was reported for discovering auto-antibodies as possible cancer markers. Tissue proteins were extracted from primary tumors, metastastic and benign tissues, and autoantigens were identified. Olfactomedin 4 (OLFM4), CD11b, integrin $\alpha 2$ (ITGA2), periostin and thrombospondin-2 were the main proteins found to be overexpressed in tumors in comparison to benign samples using a tissue microarray. These autoantigens can have prognostic significance in colorectal cancer that has a tendency to induce liver metastases. Autoantibodies can be found in the sera of patients diagnosed with colorectal cancer; thus, finding the tissue antigens that are specific for the neoplastic tissue is of outmost importance in personalizing therapy (112). When studying the association of angiogenesis-related proteins with anti-angiogenic therapy in colorectal cancer protein, arrays were used. The proteome profiler array identified in dynamics the proteins before, after treatment and after tumor progression. The antibody arrays revealed that during treatment, alterations in the levels of protein, such as MMP8, tissue inhibitor of matrix metalloproteinase (TIMP) 4 and epidermal growth factor (EGF) were observed. IL-8, Activin A and IGFBP-2, had a low association with chemotherapy induction and disease progression (113).

In organ transplantation, immunological rejection is the main clinical drawback; thus, the optimal proteomics characterization would ensure the best match between donor and recipient. Recently, a screening tool was developed using peptide array from the donor's human leukocyte antigen (HLA) to assess post-transplant sera from the recipient and evaluate the risk of immune-mediated rejection. In this pilot study, up to 600 individual peptides were customized. On these arrays pre- and post-transplant sera of recipient were investigated and, with great accuracy the immune epitopes that were involved in the immune response were detected. These personalized arrays could pin-point the donor-specific HLA epitopes and further allow the therapeutic approach to be personalized in organ transplantation (114).

In acknowledging the enhanced role of protein microarrays in research and clinical application we foresee an increased use of this technology in biomarker discovery and validation and their increased involvement in personalized medicine.

\section{Conclusions and future perspectives}

Particular proteomic-individualities of patients have recently driven the initiation of personalized medicine and these particularities can be thoroughly done only with advanced technologies (115). The Human Genome Project, has shown that there are multiple players in human disease development where the transcriptome, proteome and metabolome entered this field. However, the Human Proteome Project would never have been accomplished without the aid of several high-through-put proteomic technologies, one of these being antibody-based protein microarrays (21). New formats were constantly evolving to tackle the personalized proteome analysis. With these new formats of microarrays, parallel analyses can be carried out, investigating variations in protein structure and moreover protein interaction particular to each biological sample (116).

As cancer has a complex multifactorial trait and it is the result of acquired dysregulation at various levels (genomic, epigenomic, proteomic and metabolomics) complex multi-molecular signatures obtained from these domains would bring new information on cancer diagnosis, prognosis and personalized treatment. Platforms combining protein microarrays with bioinformatics, are bringing new tools for the further development of personalized medicine to medical and scientific communities. Protein microarrays are involved in oncology for identifying and validating new biomarkers, depicting molecules for early detection, and can monitor disease and select optimal therapeutic strategies. These platforms can intervene in all fields of oncology, as this proteomics technology can screen a multitude of parameters and encumbers tremendous future potential for applications in diagnostic and personalized medicine.

\section{Acknowledgements}

Not applicable.

\section{Funding}

This study was supported by the following grants: PN-III-P1-1.2-PCCDI-2017-0341 (PATHDERM), PNIII-P1-1.2-PCCDI-2017-0782 (REGMED) and PN 19.29.01.01.

\section{Availability of data and materials}

Not applicable.

\section{Authors' contributions}

$\mathrm{MN}, \mathrm{MB}$ and $\mathrm{CC}$ were involved in the conception and design of the manuscript, and in the writing, drafting, revising and editing of the manuscript, and in the processing of the figures and tables. All authors have read and approved the final manuscript.

\section{Ethics approval and consent to participate}

Not applicable.

\section{Patient consent for publication}

Not applicable.

\section{Competing interests}

The authors declare that they have no competing interests.

\section{References}

1. Schena M, Shalon D, Davis RW and Brown PO: Quantitative monitoring of gene expression patterns with a complementary DNA microarray. Science 270: 467-470, 1995. 
2. Gygi SP, Rochon Y, Franza BR and Aebersold R: Correlation between protein and mRNA abundance in yeast. Mol Cell Biol 19: 1720-1730, 1999.

3. Haab BB: Antibody arrays in cancer research. Mol Cell Proteomics 4: 377-383, 2005

4. Chen CS and Zhu H: Protein microarrays. Biotechniques 40: 423, 425, 427, 2006.

5. Kopf E and Zharhary D: Antibody arrays-An emerging tool in cancer proteomics. Int J Biochem Cell Biol 39: 1305-1317, 2007.

6. Constantin C, Surcel M, Munteanu A and Neagu M: Protein microarray technology for antibody detection associated to human pathology. Roman Arch Microbiol Immunol 77: 236-244, 2018.

7. Poetz O, Schwenk JM, Kramer S, Stoll D, Templin MF and Joos TO: Protein microarrays: Catching the proteome. Mech Ageing Dev 126: 161-170, 2005.

8. Speer R, Wulfkuhle J, Espina V, Aurajo R, Edmiston KH, Liotta LA and Petricoin EF III: Development of reverse phase protein microarrays for clinical applications and patient-tailored therapy. Cancer Genomics Proteomics 4: 157-164, 2007.

9. Matei C, Tampa M, Ion RM, Georgescu SR, Dumitrascu GR, Constantin $\mathrm{C}$ and Neagu M: Protein microarray for complex apoptosis monitoring of dysplastic oral keratinocytes in experimental photodynamic therapy. Biol Res 47: 33, 2014.

10. Demyanenko SV, Uzdensky AB, Sharifulina SA, Lapteva TO and Polyakova LP: PDT-induced epigenetic changes in the mouse cerebral cortex: A protein microarray study. Biochim Biophys Acta 1840: 262-270, 2014.

11. Sutandy FX, Qian J, Chen CS and Zhu H: Overview of protein microarrays. Curr Protoc Protein Sci 27: Unit 27.1, 2013.

12. Wilson JJ, Burgess R, Mao YQ, Luo S, Tang H, Jones VS, Weisheng B, Huang RY, Chen X and Huang RP: Antibody arrays in biomarker discovery. Adv Clin Chem 69: 255-324, 2015.

13. Díez P, Dasilva N, González-González M, Matarraz S Casado-Vela J, Orfao A and Fuentes M: Data analysis strategies for protein microarrays. Microarrays (Basel) 1: 64-83, 2012.

14. Angenendt P, Kreutzberger J, Glökler J and Hoheisel JD: Generation of high density protein microarrays by cell-free in situ expression of unpurified PCR products. Mol Cell Proteomics 5: 1658-1666, 2006.

15. Schweitzer B, Predki P and Snyder M: Microarrays to characterize protein interactions on a whole-proteome scale. Proteomics 3: 2190-2199, 2003.

16. Alhamdani MS, Schröder C and Hoheisel JD: Oncoproteomic profiling with antibody microarrays. Genome Med 1: 68, 2009.

17. Wulfkuhle JD, Aquino JA, Calvert VS, Fishman DA, Coukos G, Liotta LA and Petricoin EF III: Signal pathway profiling of ovarian cancer from human tissue specimens using reverse-phase protein microarrays. Proteomics 3: 2085-2090, 2003.

18. Yuk CS, Lee HK, Kim HT, Choi YK, Lee BC, Chun BH and Chung N: Development and evaluation of a protein microarray chip for diagnosis of hepatitis $\mathrm{C}$ virus. Biotechnol Lett 26 : 1563-1568, 2004.

19. Gowan SM, Hardcastle A, Hallsworth AE, Valenti MR, Hunter LJ, de Haven Brandon AK, Garrett MD, Raynaud F, Workman P, Aherne W and Eccles SA: Application of meso scale technology for the measurement of phosphoproteins in human tumour xenografts. Assay Drug Dev Technol 5: 391-401, 2007.

20. Sigalotti L, Covre A, Fratta E, Parisi G, Colizzi F, Rizzo A, Danielli R, Nicolay HJ, Coral S and Maio M: Epigenetics of human cutaneous melanoma: Setting the stage for new therapeutic strategies. J Transl Med 8: 56, 2010.

21. Honda K, Ono M, Shitashige M, Masuda M, Kamita M, Miura N and Yamada T: Proteomic approaches to the discovery of cancer biomarkers for early detection and personalized medicine. Jpn J Clin Oncol 43: 103-109, 2013.

22. Yang L, Guo S, Li Y, Zhou S and Tao S: Protein microarrays for systems biology. Acta Biochim Biophys Sin (Shanghai) 43 161-71, 2011

23. LaBaer $\mathrm{J}$ and Ramachandran N: Protein microarrays as tools for functional proteomics. Curr Opin Chem Biol 9: 14-19, 2005.

24. Hu S, Xie Z, Qian J, Blackshaw S and Zhu H: Functional protein microarray technology. Wiley Interdiscip Rev Syst Biol Med 3: 255-268, 2011.

25. He M, Stoevesandt O and Taussig MJ: In situ synthesis of protein arrays. Curr Opin Biotechnol 19: 4-9, 2008.

26. Tao SC and Zhu H: Protein chip fabrication by capture of nascent polypeptides. Nat Biotechnol 24: 1253-1254, 2006.
27. Miersch $S$ and LaBaer J: Nucleic acid programmable protein arrays: Versatile tools for array-based functional protein studies. Curr Protoc Protein Sci Chapter 27: Unit27.2, 2011.

28. Wright C, Sibani S, Trudgian D, Fischer R, Kessler B, LaBaer J and Bowness P: Detection of multiple autoantibodies in patients with ankylosing spondylitis using nucleic acid programmable protein arrays. Mol Cell Proteomics 11: M9 00384, 2012.

29. Neagu M and Constantin C: Immunogenicity of Stem Cell in Tumorigenesis Versus Regeneration. In: Stem Cells between Regeneration and Tumorigenesis. Tanase C and Neagu M (eds). Bentham Pbl. House, pp202-234, 2016.

30. Chiodoni C, Di Martino MT, Zazzeroni F, Caraglia M, Donadelli M, Meschini S, Leonetti C and Scotlandi K: Cell communication and signalling: How to turn bad language into positive one. J Exp Clin Cancer Res 38: 128, 2019.

31. James P: Protein identification in the post-genome era: The rapid rise of proteomics. Q Rev Biophys 30: 279-331, 1997.

32. Joos T and Bachmann J: Protein microarrays: Potentials and limitations. Front Biosci (Landmark Ed) 14: 4376-4385, 2009.

33. Ramachandran N, Raphael JV, Hainsworth E, Demirkan G, Fuentes MG, Rolfs A, Hu Y and LaBaer J: Next-generation high-density self-assembling functional protein arrays. Nat Methods 5: 535-538, 2008.

34. Manzano-Romána R and Fuentes M: A decade of nucleic acid programmable protein arrays (NAPPA) availability: News, actors, progress, prospects and access. J Proteomics 198: 27-35, 2019.

35. Rivera R, Wang J, Yu X, Demirkan G, Hopper M, Bian X, Tahsin T, Magee DM, Qiu J, LaBaer J and Wallstrom G: Automatic identification and quantification of extra-well fluorescence in microarray images. J Proteome Res 16: 3969-3977, 2017.

36. Yu X, Petritis B, Duan H, Xu D and LaBaer J: Advances in cell-free protein array methods. Expert Rev Proteomics 15: 1-11, 2018.

37. Yu X, Song L, Petritis B, Bian X, Wang H, Viloria J, Park J, Bui $\mathrm{H}, \mathrm{Li} \mathrm{H}$, Wang $\mathrm{J}$, et al: Multiplexed nucleic acid programmable protein arrays. Theranostics 7: 4057-4070, 2017.

38. Bulman A, Neagu M and Constantin C: Immunomics in skin cancer-improvement in diagnosis, prognosis and therapy monitoring. Curr Proteomics 10: 202-217, 2013.

39. Neagu M and Constantin C: New Insights in Cutaneous Melanoma Immune-Therapy-Tackling Immune-Suppression and Specific Anti-Tumoral Response. In: Melanoma. Murph M (ed). IntechOpen, pp225-246, 2015.

40. Butterfield LH, Ribas A, Dissette VB, Amarnani SN, Vu HT, Oseguera D, Wang HJ, Elashoff RM, McBride WH, Mukherji B, et al: Determinant spreading associated with clinical response in dendritic cell-based immunotherapy for malignant melanoma. Clin Cancer Res 9: 998-1008, 2003.

41. Yuan J, Wang E and Fox BA: Immune monitoring technology primer: Protein microarray ('seromics'). J Immunother Cancer 4: 2, 2016.

42. Chen YT, Scanlan MJ, Sahin U, Türeci O, Gure AO, Tsang S, Williamson B, Stockert E, Pfreundschuh M and Old LJ: A testicular antigen aberrantly expressed in human cancers detected by autologous antibody screening. Proc Natl Acad Sci USA 94: 1914-1918, 1997.

43. Fulton KM and Twine SM: Immunoproteomics: Current technology and applications. Methods Mol Biol 1061: 21-57, 2013.

44. Sahin U, Tureci O, Schmitt H, Cochlovius B, Johannes T, Schmits R, Stenner F, Luo G, Schobert I and Pfreundschuh M: Human neoplasms elicit multiple specific immune responses in the autologous host. Proc Natl Acad Sci USA 92: 11810-11813, 1995.

45. Ladd JJ, Chao T, Johnson MM, Qiu J, Chin A, Israel R, Pitteri SJ, Mao J, Wu M, Amon LM, et al: Autoantibody signatures involving glycolysis and splicesome proteins precede a diagnosis of breast cancer among postmenopausal women. Cancer Res 73: 1502-1513, 2013.

46. Madoz-Gurpide J, Kuick R, Wang H, Misek DE and Hanash SM: Integral protein microarrays for the identification of lung cancer antigens in sera that induce a humoral immune response. Mol Cell Proteomics 7: 268-281, 2008.

47. Bouwman K, Qiu J, Zhou H, Schotanus M, Mangold LA, Vogt R, Erlandson E, Trenkle J, Partin AW, Misek D, et al: Microarrays of tumour cell derived proteins uncover a distinct pattern of prostate cancer serum immunoreactivity. Proteomics 3: 2200-2207, 2003.

48. GuhaThakurta D, Sheikh NA, Fan LQ, Kandadi H, Meagher T, Hall SJ, Kantoff PW, Higano CS, Small EJ, Gardner TA, et al: Humoral immune response against nontargeted tumour antigens after treatment with sipuleucel-T and its association with improved clinical outcome. Clin Cancer Res 21: 3619-3630, 2015. 
49. Kwek SS, Dao V, Roy R, Hou Y, Alajajian D, Simko JP, Small EJ and Fong L: Diversity of antigen-specific responses induced in vivo with CTLA-4 blockade in prostate cancer patients. J Immunol 189: 3759-3766, 2012.

50. Graff JN, Puri S, Bifulco CB, Fox BA and Beer TM: Sustained complete response to CTLA-4 blockade in a patient with metastatic, castration-resistant prostate cancer. Cancer Immunol Res 2: 399-403, 2014.

51. Gnjatic S, Ritter E, Büchler MW, Giese NA, Brors B, Frei C, Murray A, Halama N, Zörnig I, Chen YT, et al: Seromic profiling of ovarian and pancreatic cancer. Proc Natl Acad Sci USA 107: 5088-5093, 2010.

52. Abel L, Kutschki S, Turewicz M, Eisenacher M, Stoutjesdijk J, Meyer HE, Woitalla D and May C: Autoimmune profiling with protein microarrays in clinical applications. Biomed Biochim Acta 1844: 977-987, 2014.

53. Turewicz M, May C, Ahrens M, Woitalla D, Gold R, Casjens S, Pesch B, BrüningT, Meyer HE, Nordhoff E, et al: Improving the default data analysis workflow for large autoimmune biomarker discovery studies with ProtoArrays. Proteomics 13: 2083-2087, 2013.

54. Zhang DY, Ye F, Gao L, Liu X, Zhao X, Che Y, Wang H, Wang L, $\mathrm{Wu} \mathrm{J}$, Song D, et al: Proteomics, pathway array and signaling network-based medicine in cancer. Cell Div 4: 20, 2009.

55. Schumacher S, Muekusch S and Seitz H: Up-to-date applications of microarrays and their way to commercialization. Microarrays (Basel) 4: 196-213, 2015.

56. Ayoglu B, Schwenk JM and Nilsson P: Antigen arrays for profiling autoantibody repertoires. Bioanalysis 8: 1105-1126, 2016.

57. Stoevesandt O, Taussig MJ and He M: Protein microarrays: High-throughput tools for proteomics. Expert Rev Proteomics 6 : 145-157, 2009

58. No authors listed: The call of the human proteome. Nat Methods 7: 661, 2010

59. Legrain P, Aebersold R, Archakov A, Bairoch A,Bala K, Beretta L, Bergeron J, Borchers CH, Corthals GL, Costello CE, et al: The human proteome project: Current state and future direction. Mol Cell Proteomics 10: M111.009993, 2011.

60. Jantsch MF, Quattrone A, O'Connell M, Helm M, Frye M, Macias-Gonzales M, Ohman M, Ameres S, Willems L, FuksF,etal:PositioningEurope for theEPITRANSCRIPTOMICS challenge. RNA Biol 15: 829-831, 2018.

61. González-Gomariz J, Guruceaga E, López-Sánchez M and Segura V: Proteogenomics in the context of the human proteome project (HPP). Expert Rev Proteomics 16: 267-275, 2019.

62. Tanase C, Albulescu R and Neagu M: Updates in immune-based multiplex assays. J Immunoassay Immunochem 40: 1-2, 2019.

63. Popa ML, Albulescu R, Neagu M, Eugen Hinescu ME and Tanase C: Multiplex assay for multiomics advances in personalized-precision medicine. J Immunoassay Immunochem 40: 3-25, 2019.

64. Cronin KA, Lake AJ, Scott S, Sherman RL, Noone AM, Howlader N, Henley SJ, Anderson RN, Firth AU, Ma J, et al: Annual report to the nation on the status of cancer, part I: National cancer statistics. Cancer 124: 2785-2800, 2018.

65. Smith ER: A two-tiered health care system: Is there anything new? Can J Cardiol 23: 915-916, 2007 (In English; French).

66. Alyass A, Turcotte M and Meyre D: From big data analysis to personalized medicine for all: Challenges and opportunities. BMC Med Genomics 8: 33, 2015.

67. Fröhlich H, Balling R, Beerenwinkel N, Kohlbacher O, Kumar S, Lengauer T, Maathuis MH, Moreau Y, Murphy SA, Przytycka TM, et al: From hype to reality: Data science enabling personalized medicine. BMC Med 16: 150, 2018

68. Wong SH: Pharmacogenomics and personalized medicine. In: Handbook of drug monitoring methods: Therapeutics and drugs of abuse. Dasgupta A (ed). Humana Press, New York, NY, pp211-223, 2007.

69. Yu X, Schneiderhan-Marra N and Joos TO: Protein microarrays for personalized medicine. Clin Chem 56: 376-387, 2010.

70. Masuda $M$ and Yamada T: Signalling pathway profiling by reverse-phase protein array for personalized cancer medicine. Biochim Biophys Acta 1854: 651-657, 2015.

71. Rosa M: Advances in the molecular analysis of breast cancer: Pathway toward personalized medicine. Cancer Control 22 211-219, 2015.

72. Kim DC, Wang X, Yang CR and Gao JX: A framework for personalized medicine: Prediction of drug sensitivity in cancer by proteomic profiling. Proteome Sci 10 (Suppl 1): S13, 2012.
73. Wistuba II, Gelovani JG, Jacoby JJ, Davis SE and Herbst RS: Methodological and practical challenges for personalized cancer therapies. Nat Rev Clin Oncol 8: 135-141, 2011.

74. Mueller C, Liotta L and Espina V: Reverse phase protein microarrays advance to use in clinical trials. Mol Oncol 4: 461-481, 2010.

75. Kornblau SM, Tibes R, Qiu YH, Chen W, Kantarjian HM, Andreeff M, Coombes KR and Mills GB: Functional proteomic profiling of AML predicts response and survival. Blood 113: 154-164. 2009.

76. Cain JW, Hauptschein RS, Stewart JK, Bagci T, Sahagian GG and Jay DG: Identification of CD44 as a surface biomarker for drug resistance by surface proteome signature technology. Mol Cancer Res 9: 637-647, 2011.

77. Liotta LA, Espina V, Mehta AI, Calvert V, Rosenblatt K, Geho D, Munson PJ, Young L, Wulfkuhle J and Petricoin EF III: Protein microarrays: Meeting analytical challenges for clinical applications. Cancer Cell 3: 317-325, 2003.

78. Spurrier B, Ramalingam S and Nishizuka S: Reverse-phase protein lysate microarrays for cell signaling analysis. Nat Protoc 3: 1796-1808, 2008.

79. Kim YB, Yang CR and Gao J: Functional proteomic pattern identification under low dose ionizing radiation. Artif Intell Med 49: 177-185, 2010.

80. Wang X, Dong Y, Jiwani AJ, Zou Y, Pastor J, Kuro OM, Habib AA, Ruan M, Boothman DA and Yang CR: Improved protein arrays for quantitative systems analysis of the dynamics of signaling pathway interactions. Proteome Sci 9: 53, 2011.

81. Laforte V, Lo PS, Li H and Juncker D: Antibody colocalization microarray for cross-reactivity-free multiplexed protein analysis. Methods Mol Biol 1619: 239-261, 2017.

82. Lim MS, Carlson ML, Crockett DK, Fillmore GC, Abbott DR, Elenitoba-Johnson OF, Tripp SR, Rassidakis GZ, Medeiros LJ, Szankasi P and Elenitoba-Johnson KS: The proteomic signature of NPM/ALK reveals deregulation of multiple cellular pathways. Blood 114: 1585-1595, 2009.

83. Gnjatic S, Wheeler C, Ebner M, Ritter E, Murray A, Altorki NK, Ferrara CA, Hepburne-Scott H, Joyce S, Koopman J, et al: Seromic analysis of antibody responses in non-small cell lung cancer patients and healthy donors using conformational protein arrays. J Immunol Methods 341: 50-58, 2009.

84. Gaudreau PO, Peng D, Rodriguez BL, Fradette J, Gibson L, Kundu S, Chen L, et al: Co-clinical trials of MEK inhibitor, anti PD-L1 and anti CTLA-4 combination treatment in non-small cell lung cancer. J ImmunoTherapy Cancer 6 (Suppl 1): S114, 2018.

85. Vilgelm AE, Johnson DB and Richmond A: Combinatorial approach to cancer immunotherapy: Strength in numbers. J Leukoc Biol 100: 275-290, 2016.

86. Terracciano R, Pelaia G, Preianò $M$ and Savino R: Asthma and COPD proteomics: Current approaches and future directions. Proteomics Clin Appl 9: 203-220, 2015.

87. Vizcaino JA, Csordas A, Del-Toro N, Dianes JA, Griss J, Lavidas I, Mayer G, Perez-Riverol Y, Reisinger F, Ternent T, et al: 2016 update of the PRIDE database and its related tools. Nucleic Acids Res 44: 11033, 2016.

88. Hamsten C, Häggmark A, Grundström J, Mikus M, Lindskog C, Konradsen JR, Eklund A, Pershagen G, Wickman M, Grunewald J, et al: Protein profiles of CCL5, HPGDS, and NPSR1 in plasma reveal association with childhood asthma. Allergy 71: 1357-1361, 2016.

89. Gharib SA, Nguyen EV, Lai Y, Plampin JD, Goodlett DR and Hallstrand TS: Induced sputum proteome in healthy subjects and asthmatic patients. J Allergy Clin Immunol 128: 1176-1184, e1176, 2011

90. Ohlmeier S, Nieminen P, Gao J, Kanerva T, Rönty M, Toljamo T, Bergmann U, Mazur W and Pulkkinen V: Lung tissue proteomics identifies elevated transglutaminase 2 levels in stable chronic obstructive pulmonary disease. Am J Physiol Lung Cell Mol Physiol 310: L1155-L1165, 2016.

91. Barnes PJ: Corticosteroid resistance in patients with asthma and chronic obstructive pulmonary disease. J Allergy Clin Immunol 131: 636-645, 2013.

92. Levitt JE and Rogers AJ: Proteomic study of acute respiratory distress syndrome: Current knowledge and implications for drug development. Expert Rev Proteomics 13: 457-469, 2016.

93. Priyadharshini VS and Teran LM: Personalized medicine in respiratory disease: Role of proteomics. Adv Protein Chem Struct Biol 102: 115-146, 2016. 
94. Foster MW, Morrison LD, Todd JL, Snyder LD, Thompson JW Soderblom EJ, Plonk K, Weinhold KJ, Townsend R, Minnich A and Moseley MA: Quantitative proteomics of bronchoalveolar lavage fluid in idiopathic pulmonary fibrosis. J Proteome Res 14 $1238-1249,2015$.

95. Lavoie JR, Ormiston ML, Perez-Iratxeta C, Courtman DW, Jiang B, Ferrer E, Caruso P, Southwood M, Foster WS, Morrell NW and Stewart DJ: Proteomic analysis implicates translationally controlled tumour protein as a novel mediator of occlusive vascular remodeling in pulmonary arterial hypertension. Circulation 129: 2125-2135, 2014.

96. Abdul-Salam VB, Wharton J, Cupitt J, Berryman M, Edwards RJ and Wilkins MR: Proteomic analysis of lung tissues from patients with pulmonary arterial hypertension. Circulation 122 : 2058-2067, 2010.

97. Kan M, Shumyatcher M and Himes BE: Using omics approaches to understand pulmonary diseases. Respir Res 18: $149,2017$.

98. Cohen AS, Khalil FK, Welsh EA, Schabath MB, Enkemann SA, Davis A, Zhou JM, Boulware DC, Kim J, Haura EB and Morse DL: Cell-surface marker discovery for lung cancer. Oncotarget 8: 113373-113402, 2017.

99. Monette A, Bergeron D, Ben Amor A, Meunier L, Caron C, Mes-Masson AM, Kchir N, Hamzaoui K, Jurisica I and Lapointe R: Immune-enrichment of non-small cell lung cancer baseline biopsies for multiplex profiling define prognostic immune checkpoint combinations for patient stratification. J Immunother Cancer 7: 86, 2019.

100. Schwill M, Tamaskovic R, Gajadhar AS, Kast F, White FM and Plückthun A: Systemic analysis of tyrosine kinase signaling reveals a common adaptive response program in a HER2-positive breast cancer. Sci Signal 12: eaau2875, 2019.

101. Hsu PY, Wu VS, Kanaya N, Petrossian K, Hsu HK, Nguyen D, Schmolze D, Kai M, Liu CY, Lu H, et al: Dual mTOR kinase inhibitor MLN0128 sensitizes $\mathrm{HR}^{+} / \mathrm{HER}^{+}$breast cancer patient-derived xenografts to trastuzumab or fulvestrant. Clin Cancer Res 24: 395-406, 2018.

102. Lee J, Geiss GK, Demirkan G, Vellano CP, Filanoski B, Lu Y, $\mathrm{Ju} \mathrm{Z}$, Yu S, Guo H, Bogatzki LY, et al: Implementation of a multiplex and quantitative proteomics platform for assessing protein lysates using DNA-barcoded antibodies. Mol Cell Proteomics 17: 1245-1258, 2018.

103. Kars MD and Y1ldırım G: Determination of the target proteins in chemotherapy resistant breast cancer stem cell-like cells by protein array. Eur J Pharmacol 848: 23-29, 2019.

104.Li M, Li H, Liu F, Bi R, Tu X, Chen L, Ye S and Cheng X: Characterization of ovarian clear cell carcinoma using target drug-based molecular biomarkers: Implications for personalized cancer therapy. J Ovarian Res 10: 9, 2017.

105. Mabuchi S, Sugiyama T and Kimura T: Clear cell carcinoma of the ovary: Molecular insights and future therapeutic perspectives. J Gynecol Oncol 27: e31, 2016.
106. Yanaihara N, Hirata Y, Yamaguchi N, Noguchi Y, Saito M, Nagata C, Takakura S, Yamada K and Okamoto A: Antitumor effects of interleukin-6 (IL-6)/interleukin-6 receptor (IL-6R) signalling pathway inhibition in clear cell carcinoma of the ovary. Mol Carcinog 55: 832-841, 2016

107. Yamashita Y: Ovarian cancer: New developments in clear cell carcinoma and hopes for targeted therapy. Jpn J Clin Oncol 45: 405-407, 2015.

108. Sereni MI, Baldelli E, Gambara G, Deng J,Zanotti L, Bandiera E, Bignotti E, Ragnoli M, Tognon G, Ravaggi A, et al: Functional characterization of epithelial ovarian cancer histotypes by drug target based protein signaling activation mapping: Implications for personalized cancer therapy. Proteomics 15: 365-373, 2015.

109. di Martino S, De Luca G, Grassi L, Federici G, Alfonsi R, Signore M, Addario A, De Salvo L, Francescangeli F, Sanchez M, et al: Renal cancer: New models and approach for personalizing therapy. J Exp Clin Cancer Res 37: 217, 2018.

110. Hoff FW, Hu CW, Qiu Y, Ligeralde A, Yoo SY, Mahmud H, de Bont ESJM, Qutub AA, Horton TM and Kornblau SM: Recognition of recurrent protein expression patterns in pediatric acute myeloid leukemia identified new therapeutic targets. Mol Cancer Res 16: 1275-1286, 2018.

111. Ragon BK, Odenike O, Baer MR, Stock W, Borthakur G, Patel K, Han L, Chen H, Ma H, Joseph L, et al: Oral MEK 1/2 inhibitor trametinib in combination with AKT inhibitor GSK2141795 in patients with acute myeloid leukemia with RAS mutations: A phase II study. Clin Lymphoma Myeloma Leuk S2152-2650: 31542-31548, 2019

112. Yang Q, Bavi P, Wang JY and Roehrl MH: Immuno-proteomic discovery of tumour tissue autoantigens identifies olfactomedin 4, CD11b, and integrin alpha-2 as markers of colorectal cancer with liver metastases. J Proteomics 168: 53-65, 2017.

113. Hagman H, Bendahl PO, Lidfeldt J, Belting $M$ and Johnsson A: Protein array profiling of circulating angiogenesis-related factors during bevacizumab containing treatment in metastatic colorectal cancer. PLoS One 13: e0209838, 2018.

114. Liu P, Souma T, Wei AZ, Xie X, Luo X and Jin J: Personalized peptide arrays for detection of HLA alloantibodies in organ transplantation. J Vis Exp, 2017.

115. Soni A, Gowthamarajan K and Radhakrishnan A: Personalized medicine and customized drug delivery systems: The new trend of drug delivery and disease management. Int J Pharm Compd 22: 108-121, 2018.

116. Syafrizayanti, Lueong SS, Di C, Schaefer JV, Plückthun A and Hoheisel JD: Personalised proteome analysis by means of protein microarrays made from individual patient samples. Sci Rep 7: 39756, 2017.

This work is licensed under a Creative Commons Attribution-NonCommercial-NoDerivatives 4.0 International (CC BY-NC-ND 4.0) License. 